\author{
Marquette University \\ e-Publications@Marquette
}

School of Dentistry Faculty Research and

Publications

Dentistry, School of

Fall 2015

\title{
Dental Service Utilization and Neighborhood Characteristics in Young Adults in The United States: A Multilevel Approach
}

Christopher Okunseri

Marquette University, christopher.okunseri@marquette.edu

Raul I. Garcia

Boston University

Elaye Okunseri

Marquette University, elaye.okunseri@marquette.edu

Alexis D. Visotcky

Medical College of Wisconsin

Aniko Szabo

Medical College of Wisconsin

Follow this and additional works at: https://epublications.marquette.edu/dentistry_fac

Part of the Dentistry Commons

\section{Recommended Citation}

Okunseri, Christopher; Garcia, Raul I.; Okunseri, Elaye; Visotcky, Alexis D.; and Szabo, Aniko, "Dental Service Utilization and Neighborhood Characteristics in Young Adults in The United States: A Multilevel Approach" (2015). School of Dentistry Faculty Research and Publications. 158.

https://epublications.marquette.edu/dentistry_fac/158 


\title{
Dental Service Utilization and Neighborhood Characteristics in Young Adults in The United States: A Multilevel Approach
}

\author{
Christopher Okunseri \\ School of Dentistry, Marquette University, \\ Milwaukee, WI \\ Raul I. Garcia
}

Department of Health Policy and Health Services Research, Henry M. Goldman School of Dental Medicine, Boston University, Boston, MA

Elaye Okunseri

School of Dentistry, Marquette University, Milwaukee, WI

Alexis Visotcky

Division of Biostatistics, Medical College of Wisconsin, Milwaukee, WI

Aniko Szabo

Division of Biostatistics, Medical College of Wisconsin, Milwaukee, WI 
NOT THE PUBLISHED VERSION; this is the author's final, peer-reviewed manuscript. The published version may be accessed by following the link in the citation at the bottom of the page.

\begin{abstract}
Objective: To investigate the association between neighborhood level factors and dental visits in young adults in the United States after adjusting for individual level factors.

Methods: The National Longitudinal Study of Adolescent Health Wave 1 (1994-1995) to Wave III (2001-2002) was analyzed. The primary outcome of having had at least one dental visit in the previous 12 months was analyzed via a multilevel random-effects logistic model accounting for geographic clustering in Wave III and survey design clustering from Wave I.

Neighborhood level covariates were defined at the census tract level.

Results: Overall rate of dental visits was 57 percent, highest among 18-20 year olds (65 percent) and lowest in 23-26 year olds (52 percent). Increased proportion of African-Americans ( $\leq 5$ percent to $\geq 20$ percent) and Hispanics ( $\leq 5$ percent to $\geq 20$ percent) in a neighborhood corresponded with a decrease in dental visits (60 percent versus 52 percent) and (58 percent versus 51 percent), respectively. Neighborhoods with a high proportion of collegeeducated residents had a higher percentage of dental visits. Similar differences were found when comparing the lowest and highest tertiles defined by poverty level and unemployment with dental visits. Neighborhood education was significantly associated with dental service utilization after adjustment for individual level factors and dental utilization in adolescence (Waves I and II) in the random effects model.

Conclusions: This study demonstrates that the education level of residents within a neighborhood was associated with dental service utilization in young adults in the United States.
\end{abstract}

\title{
Introduction
}

The association of person level factors such as race/ethnicity, age, gender, income, insurance, and education on health services utilization based on Andersen's behavioral model of health services ${ }^{1-4}$ has dominated the dental literature for a number of years. It is important to recognize that healthcare utilization and access to care has shifted from a strictly person-level focus to a focus on a combined mix of persons, the healthcare system, neighborhoods, and the effects of these factors on one another. $\frac{5-7}{}$ In addition, there is a growing body of research in social sciences and medicine that documents how neighborhood conditions affect self-perception of general health by influencing health behaviors, promoting diffusion of health-related information and increasing the adoption of healthy normative behaviors. $\underline{5-7}$

Neighborhood-related factors are important determinants of health services utilization and public policy. Neighborhood conditions are products of government policy, corporate investment decisions,

Journal of Public Health Dentistry, Vol 75, No. 4 (Fall 2015): pg. 282-290. DOI. This article is (C) Wiley and permission has been granted for this version to appear in e-Publications@Marquette. Wiley does not grant permission for this article to be further copied/distributed or hosted elsewhere without the express permission from Wiley. 
and political power imbalances that tend to favor some neighborhoods and harm others. In addition, neighborhoods tend to be defined by socioeconomic status and race/ethnicity, which can give rise to neighborhood health differences along similar constructs. ${ }^{8}$ One study indicated that neighborhood socioeconomic conditions have been associated with self-rated oral and general health and account for some racial/ethnic differences identified in adults. ${ }^{9}$ Sheiham and Watt reported that individual behaviors are largely determined by the conditions in which people live. ${ }^{10}$

Studies have also shown that habits formed in the earlier years of a person's lifespan tend to affect their healthcare choices in later years. ${ }^{11}$ Our study examined the relationship between neighborhood level factors and dental visits in young adults in the United States, using a multilevel approach. We expect that a better understanding of neighborhood effects in dental service utilization by young adults will be helpful for policy development and early intervention.

\section{Methods}

\section{Data source, sampling, and design}

We used data from the National Longitudinal Study of Adolescent Health (Add Health), which is the largest and most comprehensive nationally representative sample of adolescents in the United States. The database contains information on adolescents and their transition to adulthood based on three in-home interviews. Add Health used a school-based design with schools as the primary sampling units and derived the primary sampling frame from the Quality Education Database. From this frame, a stratified sample of 80 high schools (defined as schools with an 11th grade and more than 30 students) with probability proportional to size were selected. Schools were stratified by region, urbanicity, school type (public, private, and parochial), ethnic mix, and size. For each high school selected, Add health identified and recruited one of its feeder schools (typically a middle school) with probability proportional to its student contribution to the high school, yielding one school pair in each of the 80 different communities. ${ }^{12}$ More than 70 percent of the originally selected schools agreed to participate in the study. Replacement schools were selected

Journal of Public Health Dentistry, Vol 75, No. 4 (Fall 2015): pg. 282-290. DOI. This article is @ Wiley and permission has been granted for this version to appear in e-Publications@Marquette. Wiley does not grant permission for this article to be further copied/distributed or hosted elsewhere without the express permission from Wiley. 
within each stratum until an eligible school or school-pair was found. Overall, 79 percent of the schools that were contacted agreed to participate in the study. As some schools spanned grades 7-12, a total of 132 schools were in the sample, and each was associated with one of 80 communities. School size varied from fewer than 100 students to more than 3,000 students, and the communities were located in urban, suburban and rural areas of the country. ${ }^{12}$ Add Health subjects completed in-school questionnaires and a 90-minute in-home interview. Core and special supplemental samples were used. The core in-home sample is essentially self-weighting and provides a nationally representative sample of 12,105 American adolescents in grades 712.12

\section{Study design}

Our analysis was mainly based on data from Wave III (20012002) of the National Longitudinal Study of Adolescent Health (Add Health). Add Health Wave III was a follow-up interview with original Wave I respondents as they entered the transition to adulthood. ${ }^{12}$ Wave III data collection was conducted nationwide (including Hawaii and Alaska) between August 2001 and April 2002. ${ }^{12}$ Add Health completed interviews with 15,170 respondents aged 18-26 at Wave III, resulting in a 76 percent response rate. Wave III in-home interviews allowed researchers to map early trajectories out of adolescence in health and economic status and to document how adolescent experiences and behaviors are related to health outcomes in the transition to adulthood. A detailed description of the study design is available in earlier published articles. ${ }^{12,13}$

\section{Measures}

\section{Individual level variables}

The primary outcome was having had at least one dental visit in the previous 12 months as reported in Wave III. Although this study focuses on the effect of neighborhood-level factors on dental utilization, in order to minimize residual confounding, we attempted to include a wide range of individual level predictors into the model. These covariates were selected based on findings in a previous study

Journal of Public Health Dentistry, Vol 75, No. 4 (Fall 2015): pg. 282-290. DOI. This article is @ Wiley and permission has been granted for this version to appear in e-Publications@Marquette. Wiley does not grant permission for this article to be further copied/distributed or hosted elsewhere without the express permission from Wiley. 
by Okunseri etal. ${ }^{14}$ Okunseri etal. showed that in addition to demographics, dental utilization by young adults is associated with both current and adolescent socioeconomic circumstances. ${ }^{14}$

Covariates collected at the Wave III interview included age, gender, race/ethnicity, current household income, health insurance, and highest level of education. Additional covariates relevant to experiences during adolescent years were collected at Wave I. We included household income during adolescence, parental education levels, and dental utilization at Waves I and II during adolescence. Race and ethnicity were self-designated under the following categories: White, African-American, Asian or Pacific Islander, American Indian or Native American, and Other for race and having Hispanic or Latino origin versus not for ethnicity. Multiracial respondents were asked to identify a category that best described their racial background. We combined race and ethnicity into one variable and collapsed categories with low numbers to increase the stability of estimates. In addition, we placed all subjects who indicated their ethnicity as Hispanic into the Hispanic group, and participants of unknown race were included in the "Other" group. Our final groups were Whites, African Americans, Hispanics, Asians, and Other.

Household income was defined as pretax income during the previous year from all sources in the household in which the participant resided. Exact answer options offered varied between waves, so we grouped the answers into four categories that were well defined in every wave using cutoffs of $\$ 30,000, \$ 50,000$, and $\$ 75,000$ per year. Current educational attainment information was collected for respondents at Wave III, whereas information on maternal and paternal education was collected at Wave I only. As for household income, the answers were grouped to provide consistent definitions for all the educational variables. Subjects without a high school diploma or general equivalency diploma (GED) were assigned to the "less than high school" category, those with such diplomas but without a college degree (but potentially with some college-level education) were assigned to the "high school" category, those with a college degree but no postgraduate or professional degree were assigned to the "college" category, and parents/participants with a postgraduate or professional degree made up the "beyond college" category. As parental education was collected only for parents living with the adolescent, a "No resident parent" category was added to the parental education 
variables to capture such instances. Health insurance information was obtained at Wave III and was categorized as "No insurance," "Medicaid," "Private insurance," "Other insurance," or "Unknown." Information on dental insurance was not collected by Add Health and therefore not included in our analysis. History of utilization of dental services was collected at Waves I and II and recoded as having had a dental visit within the previous 12 months to mirror the primary outcome.

\section{Neighborhood level variables}

Neighborhood level covariates were defined using the 2000 census data at the census tract level. For each individual, the census tract was identified based on current residence during the Wave III interview. In parallel with the individual level covariates, we included indicators of neighborhood racial composition, education, and socioeconomic status. Racial composition was measured using two variables indicating the proportion of African American residents and proportion of residents of Hispanic origin. Neighborhood education level was quantified as the proportion of residents aged $25+$ without a high school diploma. Socioeconomic status was measured by the unemployment rate and a composite poverty index. Factor analysis was used to combine six poverty indicators: proportion of tract population, families and households under the poverty level, proportion of households receiving public assistance, proportion of female-headed households with children, and proportion of occupied households without a telephone. There was strong support for one underlying factor (Cronbach $a=0.88$ ) with approximately equal loadings. Thus, the poverty index was defined as the average of the six measures.

\section{Analytical approach}

We performed descriptive statistics and counts were reported as both the actual frequencies among the survey respondents and as weighted frequencies representing the estimated counts in the entire population from which the respondents were sampled. These are reported with standard errors. We analyzed the data via a multilevel random-effects logistic model accounting for geographic clustering in

Journal of Public Health Dentistry, Vol 75, No. 4 (Fall 2015): pg. 282-290. DOI. This article is @ Wiley and permission has been granted for this version to appear in e-Publications@Marquette. Wiley does not grant permission for this article to be further copied/distributed or hosted elsewhere without the express permission from Wiley. 
Wave III (census tracts nested within counties nested within states) and survey design clustering from Wave I. Adolescent dental utilization can be considered as intermediate outcome, mediating the effect of some of the individual and neighborhood level covariates on the primary outcome. Thus, we present analyses both including and excluding these two predictors. All analyses were adjusted for the survey design using weights that accounted for loss to follow-up from Wave I to Wave III. The analyses were performed in SAS 9.3, using the Surveyfreq procedure for descriptive statistics, and the Glimmix procedure for hierarchical logistic regression (SAS Institute, Cary, NC, USA). The effect of neighborhood-level covariates was expressed as change from the 5th to the 95th percentile in the study population to allow comparisons between effect sizes of different predictors. All model estimates are reported with 95 percent confidence intervals. The Institutional Review Boards of Marquette University and the Medical College of Wisconsin approved this study.

\section{Results}

\section{Descriptive statistics}

Table 1 represents the study population characteristics including reported dental examinations at Waves III by frequency, weighted frequencies, percent, and standard errors. A total of 5,341 participants representing 9,376,736 adolescents to young adults aged 18-26 years old in 2001-2002 participated in Wave III. In Wave III, approximately 57.4 percent of the study population reported having had a dental examination in the previous 12 months. The largest group of subjects (33 percent) was those aged $21-22$ years and the lowest (28 percent) was those 23-26 years. Slightly more females (51 percent) than males (49 percent) participated in the study and a higher proportion had a high school diploma (74 percent). Dental examination was highest among 18-20 year olds (65 percent) and lowest in 23-26 year olds (52 percent). More females (59 percent) reported having dental examinations than males. Majority of the study population were Whites (69 percent) followed by African Americans (15 percent) and Hispanics (12 percent), respectively. The groups with the highest percent of dental examination were Asians at 62 percent followed by nonHispanic Whites (61 percent).

Journal of Public Health Dentistry, Vol 75, No. 4 (Fall 2015): pg. 282-290. DOI. This article is (C) Wiley and permission has been granted for this version to appear in e-Publications@Marquette. Wiley does not grant permission for this article to be further copied/distributed or hosted elsewhere without the express permission from Wiley. 
Table 1. Descriptive Statistics for Study Population Characteristics: 20012002

\section{Predictor Frequency Weighted frequency}

Overall

Age group

18-20 years

21-22 years

23-26 years

Sex

Male

Race/ethnicity

Hispanic

African

1,585

2,104

1,652

3,007

2,334

Americans

Other

Non-Hispanic

White

\section{Education}

Beyond

college

College

85

375

739

934

82

3,211

High school

805

4,018

432

high school

Father's education

Beyond 570

college

570

College

929

High school

1,880

No resident

parent

Less than

high school

1,261

482

Mother's education

Beyond 517

college

College

1,206

High school

2,589

214

parent

Less than 639

high school

$$
\begin{aligned}
& 3,502,938 \\
& 3,484,027 \\
& 2,389,771
\end{aligned}
$$

$4,944,819$

$4,431,916$

$51.3(0.7)$

$48.7(0.7)$

336,058

929,245

$1,109,397$

$3.3(0.7)$

$11.5(1.7)$

$14.7(2.0)$

126,080

$6,875,955$

$1.5(0.3)$

$69.0(2.8)$

$1.2(0.2)$

$10.3(1.0)$

$73.6(0.9)$

$14.9(0.9)$

908,385

932,038

$1,698,445$

$3,489,536$

$15.5(1.0)$

$37.9(1.4)$

$27.6(1.3)$

$11.3(0.9)$

818,183

785,422

$2,022,468$

$6.9(0.7)$

$18.6(1.1)$

$54.5(1.4)$

$4.7(0.4)$

376,257

$1,076,126$

$15.3(1.1)$
Weighted dental exam percent (SE)

$57.4(1.2)$

$64.8(1.7)$

$55.1(1.6)$

$51.9(1.6)$

$59.0(1.2)$

55.7 (1.5)

$62.4(2.7)$

$49.5(2.2)$

46.3 (1.9)

$50.5(5.5)$

$61.0(1.3)$

$60.9(6.6)$

$70.4(2.0)$

$59.5(1.2)$

$37.4(1.7)$

$76.9(2.1)$

$70.0(1.8)$

$58.8(1.2)$

48.5 (1.5)

$46.2(2.3)$

$72.6(2.5)$

$69.4(1.6)$

$56.4(1.2)$

$51.8(3.4)$

$45.1(1.8)$

Journal of Public Health Dentistry, Vol 75, No. 4 (Fall 2015): pg. 282-290. DOI. This article is C Wiley and permission has been granted for this version to appear in e-Publications@Marquette. Wiley does not grant permission for this article to be further copied/distributed or hosted elsewhere without the express permission from Wiley. 
NOT THE PUBLISHED VERSION; this is the author's final, peer-reviewed manuscript. The published version may be accessed by following the link in the citation at the bottom of the page.

\section{Predictor Frequency Weighted frequency}

Household income

\begin{tabular}{|c|c|c|c|c|}
\hline $\begin{array}{l}\text { Less than } \\
\$ 29,999\end{array}$ & 2,541 & $4,583,678$ & $58.9(1.2)$ & $53.4(1.5)$ \\
\hline $\begin{array}{l}\$ 30,000 \text { to } \\
\$ 49,999\end{array}$ & 795 & $1,311,121$ & $16.6(0.7)$ & $54.2(1.8)$ \\
\hline $\begin{array}{l}\$ 50,000 \text { to } \\
\$ 74,999\end{array}$ & 615 & $1,091,986$ & $11.7(0.6)$ & $64.0(2.3)$ \\
\hline $\begin{array}{l}\$ 75,000 \text { or } \\
\text { more }\end{array}$ & 756 & $1,311,628$ & $12.8(0.8)$ & $70.0(1.9)$ \\
\hline \multicolumn{5}{|l|}{ Insurance } \\
\hline Medicaid & 245 & 418,613 & $6.1(0.5)$ & $42.0(2.6)$ \\
\hline None & 782 & $1,468,035$ & $24.7(0.9)$ & $36.4(1.4)$ \\
\hline Private & 4,259 & $7,381,735$ & $67.9(1.2)$ & $66.5(1.1)$ \\
\hline Unknown & 55 & 108,351 & $1.3(0.2)$ & $52.9(6.8)$ \\
\hline \multicolumn{5}{|c|}{ Wave III: tract African Americans } \\
\hline $0 \%-5 \%$ & 2,811 & $5,228,650$ & $55.0(2.6)$ & $59.7(1.3)$ \\
\hline $5 \%-20 \%$ & 1,283 & $2,136,096$ & $24.0(1.6)$ & $56.1(1.7)$ \\
\hline $20 \%-100 \%$ & 1,118 & $1,748,133$ & $21.0(2.2)$ & $52.2(2.3)$ \\
\hline \multicolumn{5}{|c|}{ Wave III: tract Hispanic } \\
\hline $0 \%-5 \%$ & 2,971 & $5,916,005$ & $64.5(2.8)$ & $57.6(1.5)$ \\
\hline $5 \%-20 \%$ & 1,240 & $2,062,669$ & $21.7(1.9)$ & $59.8(1.6)$ \\
\hline $20 \%-100 \%$ & 1,001 & $1,134,205$ & $13.9(2.0)$ & $51.3(1.9)$ \\
\hline \multicolumn{5}{|c|}{ Wave III: tract low education } \\
\hline $0 \%-13 \%$ & 1,985 & $3,734,039$ & $35.6(2.1)$ & $65.8(1.4)$ \\
\hline $13 \%-23 \%$ & 1,594 & $2,966,932$ & $33.2(1.9)$ & $56.3(1.4)$ \\
\hline $23 \%-81 \%$ & 1,633 & $2,411,908$ & $31.3(2.3)$ & $48.5(1.5)$ \\
\hline \multicolumn{5}{|c|}{ Wave III: tract poverty index } \\
\hline $0-0.07$ & 1,855 & $3,358,772$ & $32.5(2.0)$ & $64.9(1.3)$ \\
\hline $0.07-0.14$ & 1,981 & $3,369,823$ & $37.9(1.6)$ & $55.9(1.5)$ \\
\hline $0.14-0.55$ & 1,375 & $2,382,666$ & $29.6(2.1)$ & $50.5(1.6)$ \\
\hline \multicolumn{5}{|c|}{ Wave III: tract unemployment } \\
\hline $0 \%-3.5 \%$ & 1,318 & $2,550,437$ & $24.7(1.8)$ & $64.8(1.6)$ \\
\hline $3.5 \%-6.5 \%$ & 2,014 & $3,610,227$ & $38.7(1.7)$ & $58.6(1.4)$ \\
\hline $6.5 \%-87 \%$ & 1,880 & $2,952,214$ & $36.6(2.3)$ & $50.7(1.4)$ \\
\hline
\end{tabular}

\section{percent (SE) \\ Weighted}

Weighted dental exam percent (SE) 
reported dental examination decreased. Similar patterns were seen when comparing the lowest and highest tertiles defined by poverty level and unemployment.

\section{Multivariable logistic regression}

Table $\underline{2}$ shows the results for the hierarchical logistic regression models for dental examination in Wave III based on the fixed and random effects. We considered three models with successive additions of neighborhood-level predictors: first adding only racial composition, then socioeconomic status, and finally education. An additional model including all neighborhood-level predictors but not adjusting for dental service utilization during adolescence was fitted to eliminate the potential mediating effect of this intermediate outcome. After adjusting for individual level factors including sex, race/ethnicity, age, current and adolescent household income, health insurance status, self and parental education, and dental utilization in adolescence (Waves I and II) in the random effects model, neighborhood race/ethnicity composition, unemployment, and poverty were not significantly associated with dental visits in young adulthood. The educational level of the neighborhood was significantly associated with reported dental examination, with an estimated $\mathrm{OR}=0.69$ (95 percent $\mathrm{CI}$ : $0.51-0.93$ ) for comparing two hypothetical neighborhoods at the 5th and 95th percentile of residents age 25+ without high school diploma. The addition of the neighborhood-level predictors did not appreciably change the size of the individual effects as demonstrated by the essentially identical coefficients across models 1-3. Even adding the statistically significant neighborhood education level in model 3 only slightly attenuated the effect of individual education level. Omitting the potential intermediate outcomes of dental examinations during adolescence in model 4 led to a slightly stronger effect of neighborhood education and more pronounced effects of Wave I demographic characteristics. However, the results were qualitatively unchanged. 
Table 2. Hierarchical Logistic Regression Models for Dental Examination in Wave III: Fixed and Random Effects

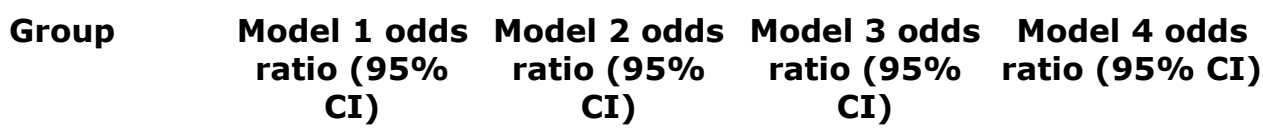

Statistical significance: "***" $<0.001 ; " * * "<0.01 ;$; $*$ " $<0.05$.

${ }^{\exists}$ Random effects are quantified as the odds ratio corresponding to moving between the 5 th and 95 th percentiles of the predictor.

Four models shown differ by which context variables are included. Model 1 demographics (proportion of African Americans, proportion of Hispanic); Model 2 demographics + SES (Poverty index, unemployment rate); Model 3 demographics + SES + education (proportion age 25+ without HS); Model 4 demographics + SES + education, no adjustment for adolescent dental examination. Fixed effects

Age group

\begin{tabular}{|c|c|c|c|c|}
\hline $16-18$ years & $\begin{array}{l}1.47(1.26- \\
1.73) * * *\end{array}$ & $\begin{array}{l}1.48(1.26- \\
1.73) * * *\end{array}$ & $\begin{array}{l}1.48(1.26- \\
1.73) * * *\end{array}$ & $\begin{array}{l}1.55(1.32- \\
1.81) * * *\end{array}$ \\
\hline $19-22$ years & $\begin{array}{l}1.14(1.00- \\
1.30)\end{array}$ & $\begin{array}{l}1.14(1.00- \\
1.30)\end{array}$ & $\begin{array}{l}1.13(0.99- \\
1.29)\end{array}$ & $\begin{array}{l}1.19(1.05- \\
1.36) * *\end{array}$ \\
\hline $23-26$ years & 1.0 (reference) & 1.0 (reference) & 1.0 (reference) & 1.0 (reference) \\
\hline \multicolumn{5}{|l|}{ Sex } \\
\hline Female & $\begin{array}{l}1.18(1.06- \\
1.33) * *\end{array}$ & $\begin{array}{l}1.18(1.06- \\
1.33)^{* *}\end{array}$ & $\begin{array}{l}1.19(1.06- \\
1.33) * *\end{array}$ & $\begin{array}{l}1.23(1.10- \\
1.38) * * *\end{array}$ \\
\hline Male & 1.0 (reference) & 1.0 (reference) & 1.0 (reference) & 1.0 (reference) \\
\hline \multicolumn{5}{|l|}{ Race/ethnicity } \\
\hline Asian & $\begin{array}{l}1.17(0.86- \\
1.58)\end{array}$ & $\begin{array}{l}1.16(0.86- \\
1.57)\end{array}$ & $\begin{array}{l}1.16(0.86- \\
1.57)\end{array}$ & $1.10(0.81-1.49)$ \\
\hline Hispanic & $\begin{array}{l}1.00(0.81- \\
1.23)\end{array}$ & $\begin{array}{l}1.00(0.81- \\
1.23)\end{array}$ & $\begin{array}{l}0.98(0.79- \\
1.20)\end{array}$ & $0.93(0.75-1.14)$ \\
\hline African Americans & $\begin{array}{l}0.78(0.64- \\
0.96)_{-}^{*}\end{array}$ & $\begin{array}{l}0.78(0.64- \\
0.96)_{-}^{*}\end{array}$ & $\begin{array}{l}0.77(0.63- \\
0.95)_{-}^{*}\end{array}$ & $\begin{array}{l}0.69(0.56- \\
0.84) * * *\end{array}$ \\
\hline Other & $\begin{array}{l}0.92(0.60- \\
1.43)\end{array}$ & $\begin{array}{l}0.93(0.60- \\
1.44)\end{array}$ & $\begin{array}{l}0.93(0.60- \\
1.43)\end{array}$ & $0.92(0.60-1.42)$ \\
\hline
\end{tabular}

Non-Hispanic White 1.0 (reference) 1.0 (reference) 1.0 (reference) 1.0 (reference) Wave I: dental examination

$\begin{array}{llll}\text { Yes } & 1.63(1.42- & 1.63(1.42- & 1.62(1.41- \\ & 1.87)_{* * *}^{* *} & 1.87)_{* * *}^{* *} & 1.85)^{* * *} \\ \text { No } & 1.0 \text { (reference) } & 1.0 \text { (reference) } & 1.0 \text { (reference) }\end{array}$

Wave II: dental examination

$\begin{array}{llll}\text { Yes } & 1.56(1.36- & 1.56(1.36- & 1.56(1.36- \\ & 1.79)^{* * *} & 1.80) * * * & 1.79)^{* * *} \\ \text { No } & 1.0 \text { (reference) } & 1.0 \text { (reference) } & 1.0 \text { (reference) }\end{array}$

Wave III: education

$\begin{array}{lllll}\text { Beyond college } & 1.69(1.04- & 1.69(1.04- & 1.63(1.00- & 1.74(1.08- \\ & 2.74)_{-}^{*} & 2.76)_{-}^{*} & 2.66)_{-}^{*} & 2.83)_{-}^{*}\end{array}$

Journal of Public Health Dentistry, Vol 75, No. 4 (Fall 2015): pg. 282-290. DOI. This article is @ Wiley and permission has been granted for this version to appear in e-Publications@Marquette. Wiley does not grant permission for this article to be further copied/distributed or hosted elsewhere without the express permission from Wiley. 
NOT THE PUBLISHED VERSION; this is the author's final, peer-reviewed manuscript. The published version may be accessed by following the link in the citation at the bottom of the page.

\begin{tabular}{|c|c|c|c|c|}
\hline Group & $\begin{array}{l}\text { Model } 1 \text { odds } \\
\text { ratio ( } 95 \% \\
\text { CI) }\end{array}$ & $\begin{array}{l}\text { Model } 2 \text { odds } \\
\text { ratio ( } 95 \% \\
\text { CI) }\end{array}$ & $\begin{array}{l}\text { Model } 3 \text { odds } \\
\text { ratio ( } 95 \% \\
\text { CI) }\end{array}$ & $\begin{array}{l}\text { Model } 4 \text { odds } \\
\text { ratio ( } 95 \% \mathrm{CI})\end{array}$ \\
\hline College & $\begin{array}{l}1.85(1.44- \\
2.38) * * *\end{array}$ & $\begin{array}{l}1.85(1.44- \\
2.38) * * *\end{array}$ & $\begin{array}{l}1.80(1.40- \\
2.32) * * *\end{array}$ & $\begin{array}{l}1.95(1.53- \\
2.50) * * *\end{array}$ \\
\hline High school & $\begin{array}{l}1.41(1.18- \\
1.70) * * *\end{array}$ & $\begin{array}{l}1.41(1.18- \\
1.70) * * *\end{array}$ & $\begin{array}{l}1.40(1.17- \\
1.68) * * *\end{array}$ & $\begin{array}{l}1.46(1.22- \\
1.75) * * *\end{array}$ \\
\hline $\begin{array}{l}\text { Less than high } \\
\text { school }\end{array}$ & 1.0 (reference) & 1.0 (reference) & 1.0 (reference) & 1.0 (reference) \\
\hline \multicolumn{5}{|l|}{ Father's education } \\
\hline Beyond college & $\begin{array}{l}1.04(0.77- \\
1.40)\end{array}$ & $\begin{array}{l}1.03(0.76- \\
1.40)\end{array}$ & $\begin{array}{l}1.01(0.74- \\
1.36)\end{array}$ & $1.13(0.84-1.52)$ \\
\hline College & $\begin{array}{l}1.00(0.78- \\
1.28)\end{array}$ & $\begin{array}{l}1.00(0.78- \\
1.28)\end{array}$ & $\begin{array}{l}0.98(0.77- \\
1.26)\end{array}$ & $1.08(0.85-1.38)$ \\
\hline High school & $\begin{array}{l}0.97(0.79- \\
1.19)\end{array}$ & $\begin{array}{l}0.97(0.79- \\
1.19)\end{array}$ & $\begin{array}{l}0.97(0.79- \\
1.19)\end{array}$ & $1.03(0.84-1.26)$ \\
\hline No resident parent & $\begin{array}{l}1.05(0.85- \\
1.29)\end{array}$ & $\begin{array}{l}1.05(0.85- \\
1.29)\end{array}$ & $\begin{array}{l}1.03(0.84- \\
1.27)\end{array}$ & $1.09(0.89-1.34)$ \\
\hline $\begin{array}{l}\text { Less than high } \\
\text { school }\end{array}$ & 1.0 (reference) & 1.0 (reference) & 1.0 (reference) & 1.0 (reference) \\
\hline \multicolumn{5}{|l|}{ Mother's education } \\
\hline Beyond college & $\begin{array}{l}1.29(0.97- \\
1.72)\end{array}$ & $\begin{array}{l}1.30(0.98- \\
1.72)\end{array}$ & $\begin{array}{l}1.26(0.95- \\
1.68)\end{array}$ & $\begin{array}{l}1.38(1.04- \\
1.82)_{-}^{*}\end{array}$ \\
\hline College & $\begin{array}{l}1.31(1.06- \\
1.64)^{*}\end{array}$ & $\begin{array}{l}1.32(1.06- \\
1.64)_{-}^{*}\end{array}$ & $\begin{array}{l}1.29(1.03- \\
1.60)^{*}\end{array}$ & $\begin{array}{l}1.37(1.11- \\
1.70) * *\end{array}$ \\
\hline High school & $\begin{array}{l}1.04(0.87- \\
1.25)\end{array}$ & $\begin{array}{l}1.04(0.87- \\
1.25)\end{array}$ & $\begin{array}{l}1.03(0.87- \\
1.24)\end{array}$ & $1.10(0.92-1.31)$ \\
\hline No resident parent & $\begin{array}{l}1.17(0.85- \\
1.62)\end{array}$ & $\begin{array}{l}1.17(0.85- \\
1.61)\end{array}$ & $\begin{array}{l}1.15(0.84- \\
1.59)\end{array}$ & $1.14(0.83-1.57)$ \\
\hline $\begin{array}{l}\text { Less than high } \\
\text { school }\end{array}$ & 1.0 (reference) & 1.0 (reference) & 1.0 (reference) & 1.0 (reference) \\
\hline \multicolumn{5}{|c|}{ Wave I: household income } \\
\hline$\$ 75,000$ or more & $\begin{array}{l}1.32(1.06- \\
1.63)^{*}\end{array}$ & $\begin{array}{l}1.31(1.06- \\
1.63)^{*}\end{array}$ & $\begin{array}{l}1.29(1.04- \\
1.60)^{*}\end{array}$ & $\begin{array}{l}1.49(1.21- \\
1.85) * * *\end{array}$ \\
\hline $\begin{array}{l}\$ 50,000 \text { to } \\
\$ 74,999\end{array}$ & $\begin{array}{l}1.32(1.11- \\
1.57) * *\end{array}$ & $\begin{array}{l}1.32(1.11- \\
1.57) * *\end{array}$ & $\begin{array}{l}1.30(1.10- \\
1.55) * *\end{array}$ & $\begin{array}{l}1.47(1.24- \\
1.74) * * *\end{array}$ \\
\hline $\begin{array}{l}\$ 30,000 \text { to } \\
\$ 49,999\end{array}$ & $\begin{array}{l}1.05(0.90- \\
1.22)\end{array}$ & $\begin{array}{l}1.05(0.90- \\
1.22)\end{array}$ & $\begin{array}{l}1.04(0.89- \\
1.21)\end{array}$ & $1.12(0.97-1.30)$ \\
\hline Less than $\$ 29,999$ & 1.0 (reference) & 1.0 (reference) & 1.0 (reference) & 1.0 (reference) \\
\hline \multicolumn{5}{|c|}{ Wave III: household income } \\
\hline$\$ 75,000$ or more & $\begin{array}{l}1.46(1.22- \\
1.75) * * *\end{array}$ & $\begin{array}{l}1.46(1.21- \\
1.76) * * *\end{array}$ & $\begin{array}{l}1.49(1.24- \\
1.79) * * *\end{array}$ & $\begin{array}{l}1.50(1.25- \\
1.81) * * *\end{array}$ \\
\hline $\begin{array}{l}\$ 50,000 \text { to } \\
\$ 74,999\end{array}$ & $\begin{array}{l}1.33(1.11- \\
1.59) * *\end{array}$ & $\begin{array}{l}1.33(1.11- \\
1.59) * *\end{array}$ & $\begin{array}{l}1.35(1.13- \\
1.62) * *\end{array}$ & $\begin{array}{l}1.33(1.11- \\
1.59) * *\end{array}$ \\
\hline $\begin{array}{l}\$ 30,000 \text { to } \\
\$ 49,999\end{array}$ & $\begin{array}{l}1.02(0.88- \\
1.18)\end{array}$ & $\begin{array}{l}1.02(0.87- \\
1.18)\end{array}$ & $\begin{array}{l}1.03(0.88- \\
1.19)\end{array}$ & $1.03(0.89-1.20)$ \\
\hline Less than $\$ 29,999$ & 1.0 (reference) & 1.0 (reference) & 1.0 (reference) & 1.0 (reference) \\
\hline
\end{tabular}

Journal of Public Health Dentistry, Vol 75, No. 4 (Fall 2015): pg. 282-290. DOI. This article is @ Wiley and permission has been granted for this version to appear in e-Publications@Marquette. Wiley does not grant permission for this article to be further copied/distributed or hosted elsewhere without the express permission from Wiley. 
NOT THE PUBLISHED VERSION; this is the author's final, peer-reviewed manuscript. The published version may be accessed by following the link in the citation at the bottom of the page.

\begin{tabular}{|c|c|c|c|c|}
\hline Group & $\begin{array}{l}\text { Model } 1 \text { odds } \\
\text { ratio ( } 95 \% \\
\text { CI) }\end{array}$ & $\begin{array}{l}\text { Model } 2 \text { odds } \\
\text { ratio ( } 95 \% \\
\text { CI) }\end{array}$ & $\begin{array}{l}\text { Model } 3 \text { odds } \\
\text { ratio ( } 95 \% \\
\text { CI) }\end{array}$ & $\begin{array}{l}\text { Model } 4 \text { odds } \\
\text { ratio ( } 95 \% \mathrm{CI})\end{array}$ \\
\hline Private & $\begin{array}{l}2.33(2.03- \\
2.68) * * *\end{array}$ & $\begin{array}{l}2.34(2.03- \\
2.68) * * *\end{array}$ & $\begin{array}{l}2.32(2.01- \\
2.66) * * *\end{array}$ & $\begin{array}{l}2.31(2.01- \\
2.65) * * *\end{array}$ \\
\hline Medicaid & $\begin{array}{l}1.38(1.06- \\
1.79)_{-}^{*}\end{array}$ & $\begin{array}{l}1.37(1.06- \\
1.79)_{-}^{*}\end{array}$ & $\begin{array}{l}1.38(1.06- \\
1.79)^{*}\end{array}$ & $\begin{array}{l}1.35(1.04- \\
1.75) *\end{array}$ \\
\hline Other/unknown & $\begin{array}{l}2.12(1.22- \\
3.69) * *\end{array}$ & $\begin{array}{l}2.13(1.22- \\
3.70) * *\end{array}$ & $\begin{array}{l}2.11(1.21- \\
3.67) * *\end{array}$ & $\begin{array}{l}1.98(1.15- \\
3.40)^{*}\end{array}$ \\
\hline \multicolumn{4}{|l|}{ Region } & 1.0 (reference) \\
\hline West & $\begin{array}{l}0.74(0.57- \\
0.97)_{-}^{*}\end{array}$ & $\begin{array}{l}0.75(0.57- \\
0.98)_{-}^{*}\end{array}$ & $\begin{array}{l}0.72(0.55- \\
0.94)_{-}^{*}\end{array}$ & $\begin{array}{l}0.69(0.52- \\
0.91) * *\end{array}$ \\
\hline Midwest & $\begin{array}{l}0.75(0.59- \\
0.95) *\end{array}$ & $\begin{array}{l}0.75(0.59- \\
0.95)_{-}^{*}\end{array}$ & $\begin{array}{l}0.74(0.58- \\
0.94)^{*}\end{array}$ & $\begin{array}{l}0.73(0.56- \\
0.94) *\end{array}$ \\
\hline South & $\begin{array}{l}0.76(0.60- \\
0.96)^{*}\end{array}$ & $\begin{array}{l}0.76(0.60- \\
0.97)_{-}^{*}\end{array}$ & $\begin{array}{l}0.77(0.61- \\
0.98)_{-}^{*}\end{array}$ & $\begin{array}{l}0.73(0.57- \\
0.93)^{*}\end{array}$ \\
\hline \multicolumn{5}{|l|}{ Random effectsa } \\
\hline $\begin{array}{l}\text { Wave III: tract } \\
\text { African Americans }\end{array}$ & $\begin{array}{l}0.95(0.75- \\
1.20)\end{array}$ & $\begin{array}{l}0.95(0.73- \\
1.25)\end{array}$ & $\begin{array}{l}0.98(0.75- \\
1.29)\end{array}$ & $1.00(0.77-1.31)$ \\
\hline $\begin{array}{l}\text { Wave III: tract } \\
\text { Hispanic }\end{array}$ & $\begin{array}{l}0.89(0.74- \\
1.08)\end{array}$ & $\begin{array}{l}0.90(0.73- \\
1.10)\end{array}$ & $\begin{array}{l}1.08(0.84- \\
1.39)\end{array}$ & $1.10(0.86-1.42)$ \\
\hline $\begin{array}{l}\text { Wave III: tract } \\
\text { poverty }\end{array}$ & - & $\begin{array}{l}1.10(0.85- \\
1.42)\end{array}$ & $\begin{array}{l}1.27(0.96- \\
1.68)\end{array}$ & $1.28(0.97-1.70)$ \\
\hline $\begin{array}{l}\text { Wave III: tract } \\
\text { unemployment }\end{array}$ & - & $\begin{array}{l}0.89(0.74- \\
1.06)\end{array}$ & $\begin{array}{l}0.89(0.74- \\
1.06)\end{array}$ & $0.88(0.74-1.05)$ \\
\hline $\begin{array}{l}\text { Wave III: tract low } \\
\text { education }\end{array}$ & - & - & $\begin{array}{l}0.69(0.51- \\
0.93)_{-}^{*}\end{array}$ & $\begin{array}{l}0.63(0.47- \\
0.86) * *\end{array}$ \\
\hline \multicolumn{5}{|l|}{ Standard deviation } \\
\hline Wave I: school ID & 0.08 & 0.08 & 0.08 & 0.11 \\
\hline Wave III: tract & 0.00 & 0.00 & 0.00 & 0.00 \\
\hline Wave III: county & 0.04 & 0.04 & 0.06 & 0.00 \\
\hline Wave III: state & 0.15 & 0.15 & 0.14 & 0.17 \\
\hline
\end{tabular}

For completeness, Table $\underline{2}$ also lists estimates of the standard deviation attributable to each level of clustering in the data: primary sampling unit and tract, county, and state of residence during young adulthood. Although inclusion of these effects is important for proper estimation of the impact of neighborhood-level predictors in however due to the specifics of the Add Health study design, these estimates are not readily interpretable. First, there is relatively little clustering of subjects at the census tract and county levels - almost 70 percent of the tracts are represented by only one person. Second, the primary sampling unit is also geographically based, and many of the subjects 
who live in the same census tract or county in Wave III are also in the same primary sampling unit. Thus, the low variability estimates at tract and county level should not be interpreted as lack of effects.

\section{Discussion}

Numerous studies on the association of neighborhood characteristics on general health in medicine, sociology, and psychology have continued to be refined based on recent theoretical approaches. $\frac{5-7,15-19}{}$ One report stated that where and with whom individuals live may directly affect their health status, health-related behavior, health needs, and health services use because of the potential impact of these variables on financial stability and stress. $\underline{20}$ This increased awareness that neighborhoods influence several health outcomes independent of person level factors has received little attention relative to dental service use. This study expands the literature on the relationship between dental service utilization and neighborhood characteristics of young adults in the United States.

First, we examined the prevalence of dental examinations in young adults as well as the distribution by different demographic factors. We found that over 50 percent of young adults reported having had a dental examination. Although this finding potentially relates to individual level factors, it is important to note that individual oral health is not only influenced by individual characteristics, but also by contextual characteristics. Leyland et al. indicated that neighborhood influences act either directly or indirectly by influencing individual behavior or coping strategies. $\underline{21}$

Second, we examined the distribution of dental examinations in young adults by different demographic factors. We found that the percentage of individuals with a dental examination was highest among 18-20 year olds and lowest among 23-26 year olds. This finding is somewhat consistent with that reported by the Medical Expenditure Panel survey, which indicated that over 50 percent of adolescents up aged $\leq 20$ years had at least one dental visit in the year 2004 and that this remained virtually unchanged from the 51 percent reported in 1996. $\underline{22,23}$ In our analysis, the percentage of females with dental examination was higher than that of males. This finding is consistent with what has been reported in the literature for many

Journal of Public Health Dentistry, Vol 75, No. 4 (Fall 2015): pg. 282-290. DOI. This article is @ Wiley and permission has been granted for this version to appear in e-Publications@Marquette. Wiley does not grant permission for this article to be further copied/distributed or hosted elsewhere without the express permission from Wiley. 
NOT THE PUBLISHED VERSION; this is the author's final, peer-reviewed manuscript. The published version may be accessed by following the link in the citation at the bottom of the page.

years $\underline{24,25}$ and reflects what is often reported and seen in clinical settings.

Third, we recognize that individual characteristics only explain some of the individual differences identified by demographic factors and that neighborhoods could influence the healthcare system and health. $\underline{26}$ Neighborhood characteristics, such as poverty, racial/ethnic composition, and educational attainment, which can be obtained from US Census estimates, have hitherto received limited attention from dentistry. We investigated the association of neighborhood characteristics (e.g., education level, unemployment rate, proportion of African Americans or Hispanics, and poverty rate) on dental visits in young adults in the United States after adjusting for individual level factors. In our study, we found that after taking into account individual characteristics in the random effect model, only the educational level of a neighborhood was significantly associated with reporting a dental examination. This finding reflects the fact that neighborhood environments may be associated with the use of dental services through a variety of mechanisms. One simple mechanism could be differences in the educational levels of residents in a particular neighborhood. Neighborhoods provide a place for social interaction, exchange of cultural values, and development of positive oral health habits. It is important to note that dental public health and health promotion activities occur in individuals within their neighborhood. Our findings suggest a need for continuous health education and promotion activities to help sustain or improve upon the dental service utilization of young adults in their neighborhood.

Another interesting finding in our descriptive statistics was that as the proportion of subjects without a high school diploma in a neighborhood increased, there was a corresponding decrease in the proportion of subjects with reported dental examinations. Similar patterns were identified when comparing the lowest and highest tertiles defined by poverty level and unemployment. Tellez etal. examined the relationship between neighborhoods and the severity of dental caries among low-income African Americans and concluded that neighborhoods contribute something unique to caregivers' oral health, beyond socioeconomic position and individual risk factors. $\underline{27}$ Turrell et al. examined the association between neighborhood disadvantage and individual-level socioeconomic position on self-reported oral health

Journal of Public Health Dentistry, Vol 75, No. 4 (Fall 2015): pg. 282-290. DOI. This article is @ Wiley and permission has been granted for this version to appear in e-Publications@Marquette. Wiley does not grant permission for this article to be further copied/distributed or hosted elsewhere without the express permission from Wiley. 
in adults age 43-57 in Australia and concluded that the socioeconomic characteristics of neighborhoods are important for oral health over and above the socioeconomic characteristics of the people living in those places. $\underline{28}$

Neighborhood characteristics including socioeconomic conditions have been associated with self-rated oral and general health and account for some of the racial/ethnic differences identified in adults. 28 Neighborhoods tend to follow a pattern along lines of socioeconomic status and race/ethnicity, 9 and this pattern gives rise to neighborhood health differences and service utilization along similar lines. In our study, we did not find a significant association between having a dental examination and neighborhood levels of unemployment, poverty status, and race/ethnicity after adjusting for individual predictors. This finding is not consistent with literature evidence. $\underline{29,30}$ However, we believe that the differences between these studies could be related to differences in the research question, analytic approach, study design such as the actual questions for data collection, as well as the unique ability of this study to adjust for predictors from adolescence.

Certain potential limitations of our study should be noted. First, the study used self-reported dental examination information, and the responses from the survey could not be validated. Second, a schoolbased design was used with schools as the primary sampling units. This could limit the opportunity to gather information from other young adults who were not adolescents in the schools at the time of data collection and could possibly limit the generalizability of our findings to all young adults. Additionally, participants who lived in the same neighborhood during young adulthood often belong to the same school-based primary sampling unit, thus direct estimates of variability are confounded. There was also a lack of information about whether respondents had dental insurance. Finally, omitted variable bias is another possible limitation in our study. In conclusion, our study demonstrates that the education level of residents within a neighborhood was associated with dental service utilization among young adults in the United States. Understanding oral health behaviors of young adults and their relationships to neighborhoods is important for early intervention as well as for program and policy development.

Journal of Public Health Dentistry, Vol 75, No. 4 (Fall 2015): pg. 282-290. DOI. This article is (C Wiley and permission has been granted for this version to appear in e-Publications@Marquette. Wiley does not grant permission for this article to be further copied/distributed or hosted elsewhere without the express permission from Wiley. 
NOT THE PUBLISHED VERSION; this is the author's final, peer-reviewed manuscript. The published version may be accessed by following the link in the citation at the bottom of the page.

\section{Acknowledgments}

This research uses data from Add Health, a program project directed by Kathleen Mullan Harris and designed by J. Richard Udry, Peter S. Bearman, and Kathleen Mullan Harris at the University of North Carolina at Chapel Hill, and funded by grant P01-HD31921 from the Eunice Kennedy Shriver National Institute of Child Health and Human Development, with cooperative funding from 23 other federal agencies and foundations. Special acknowledgment is due Ronald R. Rindfuss and Barbara Entwisle for assistance in the original design. Information on how to obtain the Add Health data files is available on the Add Health website (http://www.cpc.unc.edu/addhealth). No direct support was received from grant P01-HD31921 for this analysis.

Project grant support: This work was supported by a grant from the National Institute of Health; grant\#R03DE021676.

Conflicts of interest: The authors report that there are no known conflicts of interest.

Author contributions: C. Okunseri and A. Szabo conceived the study and obtained research funding. A. Szabo and A. Visotcky provided statistical advice on the study design, analyzed the data, and contributed to the methods section. C. Okunseri, A. Szabo and R. Garcia supervised the study. C. Okunseri and E. Okunseri provided research support, drafted the manuscript, and all authors contributed substantially to interpretation of results and revision of all drafts. C. Okunseri takes responsibility for the paper as a whole.

\section{References}

${ }^{1}$ Goldsmith L. A critical history of Andersen's Behavioral Model of Health Services Use: a reflection of how we study access to health care. Abstr Acad Health Ser Res Health Policy Meet. 2002;19:6.

${ }^{2}$ Andersen R, Newman JF. Societal and individual determinants of medical care utilization in the United States. Milbank Mem Fund $Q$ Health Soc. 1973;51(1):95-124.

${ }^{3}$ Yu SM, Bellamy HA, Schwalberg RH, Drum MA. Factors associated with use of preventive dental and health services among U.S. adolescents. J Adolesc Health. 2001;29:395-405.

Journal of Public Health Dentistry, Vol 75, No. 4 (Fall 2015): pg. 282-290. DOI. This article is @ Wiley and permission has been granted for this version to appear in e-Publications@Marquette. Wiley does not grant permission for this article to be further copied/distributed or hosted elsewhere without the express permission from Wiley. 
${ }^{4}$ Macek MD, Edelstein BL, Manski RJ. An analysis of dental visits in U.S. children, by category of service and sociodemographic factors, 1996. Pediatr Dent. 2001;23(5):383-389.

${ }^{5}$ Macintyre S, Ellaway A, Cummins S. Place effects on health: how can we conceptualise, operationalise and measure them? Soc Sci Med. 2002;55:125-139.

${ }^{6}$ Krieger N, Chen JT, Waterman PD, Soobader MJ, Subramanian SV, Carson R. Choosing area-based socioeconomic measures to monitor social inequalities in low birthweight and childhood lead poisoning - the Public Health Disparities Geocoding Project (US). J Epidemiol Community Health. 2003;57:186-199.

${ }^{7}$ Krieger N, Zierler S, Hogan JW, Waterman P, Chen J, Lemieux K, Gjelsvik A. Geocoding and measurement of neighborhood socioeconomic position. In: Kawachi I, Berkman LF, editors. Neighborhoods and health. New York: Oxford University Press; 2003. p. 147-178.

${ }^{8}$ Krieger N, Waterman PD, Chen JT, Soobader M-J, Subramanian SV. Monitoring socioeconomic inequalities in sexually transmitted infections, tuberculosis, and violence: geocoding and choice of area-based socioeconomic measures - the Public Health Disparities Geocoding Project (US). Public Health Rep. 2003; 118:240-260.

${ }^{9}$ Borrell LN, Taylor GW, Borgnakke WS, Woolfolk MW, Nyquist LV. Perception of general and oral health in white and African American adults; assessing the effect of neighborhood socioeconomic conditions. Community Dent Oral Epidemiol. $2004 ; 32: 363-373$

${ }^{10}$ Sheiham A, Watt R. Improving oral health for all: focusing on determinants and conditions. Health Educ J. 2000;59:64-79.

${ }^{11}$ Halfon N, Hochstein M. Life course health development: an integrated framework for developing health, policy and research. Milbank Q. 2002;80(30):1-31.

${ }^{12}$ Kathleen $\mathrm{MH}$ Design features of add health. Carolina Population Center, University of North Carolina at Chapel Hill 2007 Oct [cited 2009 Aug 20]. Available from: http://www.asanet.org/cs/root/leftnav/publications/journals/soc ial psychology quarterly/add health

${ }^{13}$ Harris KM, Halpern CT, Whitsel E, Hussey J, Tabor J, Entzel P, Udry JR. The National Longitudinal Study of Adolescent to Adult

Journal of Public Health Dentistry, Vol 75, No. 4 (Fall 2015): pg. 282-290. DOI. This article is (C) Wiley and permission has been granted for this version to appear in e-Publications@Marquette. Wiley does not grant permission for this article to be further copied/distributed or hosted elsewhere without the express permission from Wiley. 
Health: Research Design; 2009 [WWW document]. Available from: http://www.cpc.unc.edu/projects/addhealth/design

${ }^{14}$ Okunseri C, Okunseri E, Garcia RI, Visotcky A, Szabo A. Predictors of dental care use: findings from the National Longitudinal Study of Adolescent Health. J Adolesc Health. 2013;53:663-670.

${ }^{15}$ Diez-Roux AV. Investigating neighborhood and area effects on health. Am J Public Health. 2001;91:1783-1789.

${ }^{16}$ Rajaratnam JK, Burke JG, O'Campo P. Maternal and child health and neighborhood context: The selection and construction of arealevel variables. Health Place. 2006;12:547-556.

${ }^{17}$ Diez Roux AV. The examination of neighborhood effects on health: conceptual and methodological issues related to the presences of multiple levels of organization. In: Kawachi I, Berkman LF, editors. Neighborhoods and health. New York: Oxford University Press; 2003. p. 20-44, .

${ }^{18}$ Tienda M. Poor people and poor places: deciphering neighborhood effects on poverty outcomes. In: Huber J, editor. Macro-micro linkages in sociology. Newbury Park: Sage; 1991. p. 244-262.

${ }^{19}$ Pickett KE, Pearl M. Multilevel analyses of neighborhood socioeconomic context and health outcomes: a critical review. J Epidemiol Community Health. 2001;55:111-122. doi: 10.1136/jech.55.2.111.

${ }^{20}$ National Research Council and Institute of Medicine. Adolescent health services: missing opportunities. Committee on Adolescent Health Care Services and models of care for treatment, prevention, and health development. In: Lawrence RS, Gootman JA, Sim LJ, editors. Board on children, youth, and families. Division of behavioral and social sciences and education. Washington, DC: The National Academies Press; 2009.

${ }^{21}$ Leyland $\mathrm{AH}$, Groenewegen PP. Multilevel modelling and public health policy. Scand J Public Health. 2003;31:267-274.

${ }^{22}$ Manski RJ, Brown E. Dental use, expenses, private dental coverage, and changes, 1996 and 2004. Rockville, MD: Agency for Healthcare Research and Quality; 2007. [cited 20 Aug 2012] Available from: http://www.meps.ahrq.gov/mepsweb/data files/publications/cb 17/cb17.pdf. MedEPS Chartbook No.17.

${ }^{23}$ US Department of Health and Human Services. Oral health in America: a report of the surgeon general. Rockville, MD: US

Journal of Public Health Dentistry, Vol 75, No. 4 (Fall 2015): pg. 282-290. DOI. This article is (C) Wiley and permission has been granted for this version to appear in e-Publications@Marquette. Wiley does not grant permission for this article to be further copied/distributed or hosted elsewhere without the express permission from Wiley. 
Department of Health and Human Services, National Institute of Dental and Craniofacial Research, National Institutes of Health; 2000.

${ }^{24}$ Macek MD, Manski RJ, Vargas CM, Moeller JF. Comparing oral health care utilization estimates in the United States across three nationally representative surveys. Health Serv Res. 2002;37(2):499-521.

${ }^{25}$ Okunseri C, Pajewski NM, McGinley EL, Hoffmann RG. Racial/ethnic disparities in self-reported pediatric orthodontic visits in the United States. J Public Health Dent. 2007;67:217-223.

${ }^{26}$ Macintyre S, Ellaway A, Cummins S. Place effects on health: how can we conceptualise, operationalize and measure them? Soc Sci Med. 2002;320:1200-1204.

${ }^{27}$ Tellez M, Sohn W, Burt BA, Ismail AI. Assessment of the relationship between neighborhood characteristics and dental caries severity among low-income African-Americans: a multilevel approach. $J$ Public Health Dent. 2006;66(1):30-36.

${ }^{28}$ Turrell G, Sanders AE, Slade GD, Spencer AJ, Marcenes W. The independent contribution of neighborhood disadvantage and individual-level socioeconomic position to self-reported oral health: a multilevel analysis. Community Dent Oral Epidemiol. 2007;35(3):195-206.

${ }^{29}$ Jencks C, Mayer SE. The social consequences of growing up in a poor neighborhood. In: Lynn L Jr, McGeary MGH, editors. Inner-city poverty in the United States. Washington, DC: The National Academics Press; 1990. p. 111-186.

${ }^{30}$ Atkins $R$, Sulik MJ, Hart D. The association of individual characteristics and neighborhood poverty on the dental care of American adolescents. J Public Health Dent. 2012;72(4):313319. been granted for this version to appear in e-Publications@Marquette. Wiley does not grant permission for this article to be further copied/distributed or hosted elsewhere without the express permission from Wiley. 